\author{
Alexander M. Korsunsky \\ Department of Engineering Science, \\ University of Oxford, \\ Parks Road, \\ Oxford 0X1 3PJ, UK \\ e-mail: alexander.korsunsky@eng.ox.ac.uk
}

\title{
Residual Elastic Strains in Autofrettaged Tubes: Elastic-Ideally Plastic Model Analysis
}

Autofrettage is a treatment process that uses plastic deformation to create a state of permanent residual stress within thick-walled tubes by pressurizing them beyond the elastic limit. The present paper presents explicit analytical formulas for residual elastic strains within the tube wall derived on the basis of the classical elastic-ideally plastic solution. Then the problem is addressed of rational interpretation of the radial and hoop residual elastic strains measured at a fixed number of points. To this end, the mismatch between the experimental measurements and theoretical predictions of the residual elastic strains is represented in the form of quadratic functional, $J$, the minimum of which is sought in terms of the problem parameters, namely, the material yield stress, $\sigma_{Y}$, and the radial position of the elastic-plastic boundary, c. It is shown that J shows an approximately parabolic variation in terms of either parameter when the other is fixed, and that therefore the global minimum of $J$ can be readily found. This procedure is implemented and applied to a set of experimental data on neutron diffraction measurements (Venter, A.M., de Swardt, R.R., and Kyriacou, S., 2000, J. Strain Anal., 35, pp. 459-469). In conclusion, further applications of this family of interpretation approaches are discussed. [DOI: 10.1115/1.2400267]

\section{Introduction}

The broad context of the present study is the interpretation of experimental measurements of residual elastic strains (or their changes) with the purpose of reconstructing the complete residual stress field and of identifying the underlying inelastic strain distribution that acts as the source of the residual stress state. More specifically, the present study addresses the first of the two above questions in the context of the analysis of axially symmetric inelastic deformation of thick-walled tubes subjected to internal pressure exceeding the elastic limit of the tube. As the pressure is increased, it induces plastic deformation within a hollow cylinder with the external boundary that progresses outward from the inner bore toward the outer surface of the tube.

The analysis of the elastic stresses and strains within a thickwalled hollow cylinder is a classical problem within the theory of elasticity associated with the name of Lamé [1]. The elasticideally plastic solution can be found in Den Hartog [2], Hill [3], and Soutas-Little [4]. The complete residual elastoplastic stress and strain state can be written down analytically, making this problem a textbook case of residual stress analysis. Closed-form solutions for internally pressurized thick-walled cylinders obeying elastic-strain-hardening plastic material constitutive law were presented by Gao and Wei [5] for plane strain and Gao et al. [6] for plane stress cases. The strain gradient solution was also covered in [7].

In the present paper, we use the simplest elastic-ideally plastic formulation and quote the complete set of formulas for the residual elastic strains and the stresses explicitly for the purposes of constructing the inverse problem formulation. This approach is used to provide the basis for rational interpretation of the experimental residual elastic strain data published by Venter et al. [8]. To this end, a new variational procedure is proposed based on the

Contributed by the Materials Division of ASME for publication in the JournaL of Engineering Materials and Technology. Manuscript received September 10, 2005; final manuscript received June 5, 2006. Review conducted by Somnath Ghosh. representation of the mismatch between the model prediction and the experiment in the form of a quadratic functional that lends itself naturally to the task of finding the best model parameters by minimization. The entire procedure is implemented within MATHEMATICA $^{\circledR}$ and furnishes results within seconds. When the best values of the problem parameters are found, the complete residual elastic field can be readily reconstructed. In the conclusion, the wider context for the application of this approach is discussed.

\section{Direct Problem of Autofrettage}

The solution for elastic axisymmetric deformation of a thickwalled tube of internal radius $a$ and external radius $b$ under internal pressure $p$ is a special case of the Lamé problem (Fig. 1). The following formulas give the radial stress $\sigma_{r r}$ and hoop stress $\sigma_{\theta \theta}$ [2]:

$$
\sigma_{r r}^{0}(r)=-p \frac{\left(b^{2}-r^{2}\right) a^{2}}{\left(b^{2}-a^{2}\right) r^{2}}, \quad \sigma_{\theta \theta}^{0}(r)=p \frac{\left(b^{2}+r^{2}\right) a^{2}}{\left(b^{2}-a^{2}\right) r^{2}}
$$

Now assume that the internal pressure $p$ is sufficient to cause plastic yielding in the inner annulus of the tube, $a<r<c$, whereas the outer part of the tube, $c<r<b$, remains elastic. The solution can then be split into two parts by assuming that the radial traction transmitted across the cylindrical boundary $r=c$ is given by the as yet unknown pressure $q$ (assumed to be a positive number for convenience). Because of the problem's symmetry the shear tractions vanish everywhere, $\sigma_{r \theta}=\sigma_{r z}=0$, and the following boundary conditions apply:

$$
\begin{gathered}
\text { Inner plastic region: } \sigma_{r r}(a)=-p, \quad \sigma_{r r}(c)=-q \\
\text { Outer elastic region: } \sigma_{r r}(c)=-q, \quad \sigma_{r r}(b)=0
\end{gathered}
$$

The solution for the outer elastic region can then be readily found by modification of Eqs. (1) using the following substitutions:

$$
p \rightarrow q, \quad a \rightarrow c
$$




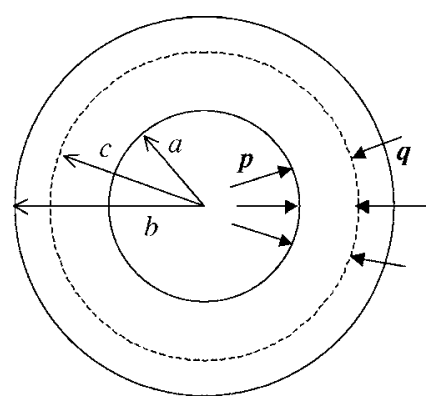

Fig. 1 Schematic illustration for the description of axisymmetric deformation of a thick-walled tube of internal radius $a$ and external radius $b$ under internal pressure $p$. Parameter $c$ indicated the radius of the elastic-plastic boundary, and $q$ is the pressure transmitted across this interface.

$$
\sigma_{r r}^{e}(r)=-q \frac{\left(b^{2}-r^{2}\right) c^{2}}{\left(b^{2}-c^{2}\right) r^{2}}, \quad \sigma_{\theta \theta}^{e}(r)=q \frac{\left(b^{2}+r^{2}\right) c^{2}}{\left(b^{2}-c^{2}\right) r^{2}}, \quad c \leq r \leq b
$$

The solution for the plastic region is developed as follows. Using Tresca's criterion, it is assumed that everywhere within the plastic region the following yield conditions is satisfied:

$$
\sigma_{\theta \theta}-\sigma_{r r}=\sigma_{Y}
$$

where $\sigma_{Y}$ is the yield stress in uniaxial tension. This equation can be solved together with the radial equilibrium equation

$$
\frac{\partial \sigma_{r r}}{\partial r}+\frac{\sigma_{r r}-\sigma_{\theta \theta}}{r}=0
$$

Satisfying the first of the boundary conditions (2) gives the following expressions for stresses in the inner plastic region [2]:

$$
\sigma_{r r}^{p}=\sigma_{Y} \log \frac{r}{a}-p, \quad \sigma_{\theta \theta}^{p}=\sigma_{Y}\left(\log \frac{r}{a}+1\right)-p, \quad a \leq r \leq c
$$

Using the second of the boundary conditions (2), an expression for $q$ is obtained

$$
q=p-\sigma_{Y} \log \frac{c}{a}
$$

Finally, noting that the yield condition (6) must also be satisfied at the boundary $r=c$ of the outer elastic region, the implicit equation for the extent of the plastic zone $c$ is obtained in terms of the problem parameters

$$
p=\frac{\sigma_{Y}}{2}\left(1-\frac{c^{2}}{b^{2}}+2 \log \frac{c}{a}\right)
$$

Equations (8) and (5) together provide the complete stress solution for the problem of elastoplastic deformation of a thick-walled tube under internal pressure.

In order to obtain the expressions for residual stresses and residual elastic strains, we now consider the process of elastic unloading. The stresses given by Eqs. (8) and (5) are modified by subtracting the solution for purely elastic stresses given in Eq. (1), so that the final residual stress state has the form

$$
\begin{array}{ccc}
\sigma_{r r}^{R}=\sigma_{r r}^{p}-\sigma_{r r}^{0}, & \sigma_{\theta \theta}^{R}=\sigma_{\theta \theta}^{p}-\sigma_{\theta \theta}^{0}, & a \leq r \leq c \\
\sigma_{r r}^{R}=\sigma_{r r}^{e}-\sigma_{r r}^{0}, & \sigma_{\theta \theta}^{R}=\sigma_{\theta \theta}^{e}-\sigma_{\theta \theta}^{0}, & c \leq r \leq b
\end{array}
$$

Assuming plane stress conditions, the residual elastic strains can be written in the form

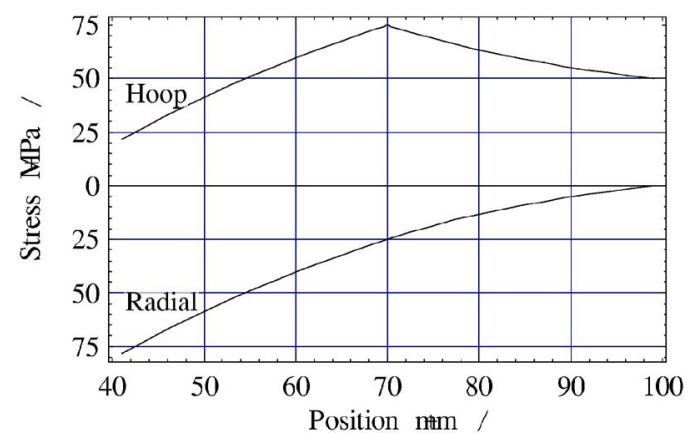

Fig. 2 Elastic-ideally plastic model predictions for stress distributions under maximum internal pressure loading

$$
E e_{r r}\left(r, c, p, a, b, \sigma_{Y}\right)=\sigma_{r r}^{R}-\nu \sigma_{\theta \theta}^{R}, \quad E e_{\theta \theta}\left(r, c, p, a, b, \sigma_{Y}\right)=\sigma_{\theta \theta}^{R}-\nu \sigma_{r r}^{R}
$$

Figures 2-4 present examples of the distributions of stresses and strains arising from the model. Some particular values of the model parameters are chosen here, although this choice is not significant at this point, since the figures are only meant to provide an illustration.

Figure 2 shows the hoop and radial stress distribution with radial position at maximum loading. The hoop stress reaches a peak value at $r=70 \mathrm{~mm}$, that in this illustrative example corresponds to the position of the elastic-plastic boundary $c$. Figure 3 illustrates the residual stresses after unloading. Figure 4 shows the residual elastic strains.

The formulas given in this section provide complete the solution of the direct problem about the determination of residual elastic strains from given geometry $(a, b)$, material properties $\left(\sigma_{Y}\right.$, Young's modulus $E$ and Poisson's ratio $\nu$ ), and loading conditions (pressure $p$ ). In other words, the residual elastic strain profiles $\varepsilon_{r r}$ and $\varepsilon_{\theta \theta}$, in a rather general sense, can be thought of as functions

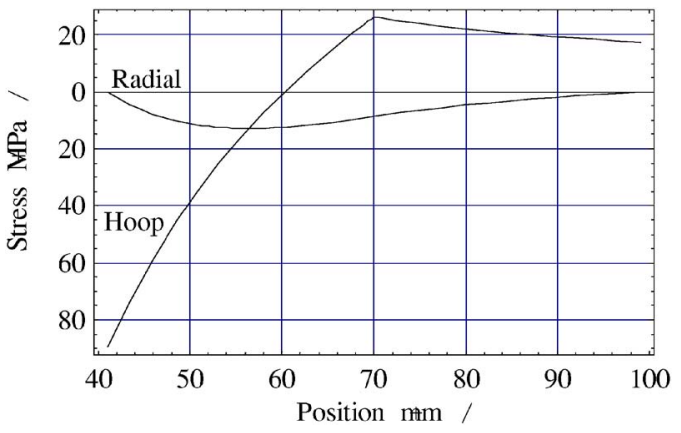

Fig. 3 Elastic-ideally plastic model predictions for stress distributions after unloading

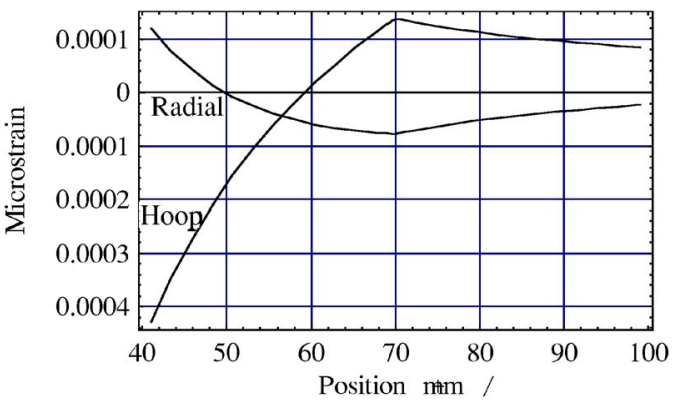

Fig. 4 Elastic-ideally plastic model predictions for residual elastic strains 


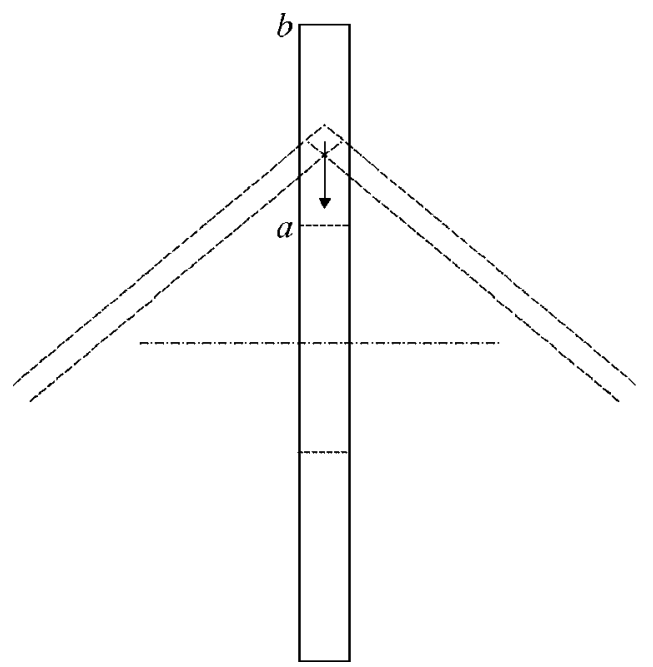

Fig. 5 A possible arrangement of autofrettaged tube slices with respect to the incident and diffracted beams. The dashed lines indicate the incident and diffracted beams; the arrow shows the scattering vector indicates the orientation of the strain component being measured (radial in the present example).

of the problem parameters $c, p, a, b, \sigma_{Y}$. This view provides a basis for developing an approach to solving the inverse problem of autofrettage (Sec. 3). However, first the experimental data from neutron diffraction measurements must be introduced in Sec. 3 .

\section{Description of Experimental Data}

Experimental results of strain evaluation in slices obtained from autofrettaged tubes are obtained and described by Venter et al. [8], who used three different techniques: neutron diffraction, Sachs boring [9], and the compliance method [10]. We focus particular attention on the neutron diffraction method, since the authors of that paper conclude that this method provides the most detailed and reliable assessment of the residual elastic strains.

Strain evaluation by diffraction is achieved by monitoring the shift in the position of the scattered peak center. This can be done either in the angular dispersive configuration, by scanning the scattering angle $2 \theta$ from a monochromatic beam; or in the energy dispersive mode, by monitoring the energy of scattered photons using an energy-resolving detector and a white beam.

It is then possible to deduce the lattice orientation specific elastic strain using Bragg's law

$$
2 d_{h k l} \sin \theta=\lambda=\frac{h c}{E}
$$

using the following formula:

$$
\varepsilon_{h k l}=\frac{d_{h k l}-d_{h k l}^{0}}{d_{h k l}^{0}}
$$

The selection of the most appropriate diffraction peak is a separate research topic in experimental strain analysis. Here we content ourselves with following the conclusions of Venter et al. [8] that the (211) peak in steel presents the best choice for the purposes of estimating the engineering macroscopic average strain.

The experimental data of Venter et al. [8] were collected using monochromatic neutron diffraction on the residual strain instrument of the SAFARI-1 research reactor operated by NECSA in Pretoria, South Africa.

Figure 5 illustrates a possible arrangement and indicates the positions of the incident and diffracted beams. The diffraction peak was obtained from the scattering volume occupying a cube of approximately $4 \mathrm{~mm}$ side length, thus providing significant av-

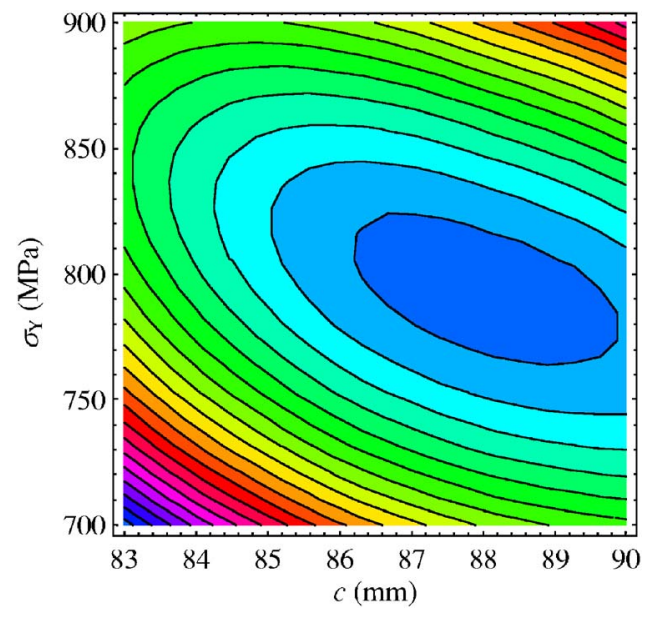

Fig. 6 The comparison between the measured and predicted residual elastic strains (experimental data of [8]). Continuous curves correspond to the predictions, whereas the markers indicate experimental results.

eraging. The markers in Fig. 6 indicate the measured values of radial and hoop residual elastic strains, respectively. Qualitative consideration of the profiles allows ready approximate identification of the position of the plastic zone, by comparison to the elastic-ideally plastic model predictions shown in Figs. 2-4. However, we seek to construct a rational procedure for more accurate identification of the problem parameters, as introduced in Sec. 4.

\section{Elastoplastic Inverse Problem Formulation}

The problem that we wish to address in the present study stands in an inverse relationship to the one solved in Sec. 2. In practice, it is the residual elastic strain distribution that may become known, e.g., from diffraction measurement, and the details of the deformation process need to be found, such as the depth of the plastic zone. Alternatively, in the absence of nondestructive measurements of residual elastic strain, changes in the elastic strain can be monitored, e.g., using strain gages, in the course of material removal. In all cases, the purpose is to determine the unknown parameters of the deformation.

In practice, the residual elastic strain, or its increments, can only be measured at a finite number of points. We are therefore seeking to find the unknown deformation parameters, such as the yield stress $\sigma_{Y}$ and the plastic zone outer radius $c$ by matching the residual elastic strain distributions predicted by the elastoplastic model of Sec. 2, to the finite data set of measured values.

Several questions arise in this situation. Can the problem be inverted? Is the inverse problem regular, i.e., does it vary smoothly depending on the unknown parameters? Would the obtained solution be unique? Although we do not attempt to answer these questions here, we construct an inversion procedure that can subsequently be evaluated in terms of its uniqueness and regularity.

Consider a set of experimental data consisting of the values of radial residual elastic strains (RES) $\varepsilon_{r r}^{j}$ and hoop residual elastic strains (RES) $\varepsilon_{\theta \theta}^{j}$ collected at positions $r_{j}, j=1, \ldots, m$. Thus, we assume that the data were collected from a one-dimensional scan in coordinate $r$, but that two components of residual elastic strain were measured at each point. It is worth noting, however, that the approach presented below is not in any way limited to problems arising from one-dimensional scans and can be readily generalized to two- and three-dimensional cases.

Now denote by $e_{r r}(r)$ and $e_{\theta \theta}(r)$ the predicted, or modeled distributions of, respectively, the radial and hoop components of the residual elastic strain. Evaluating $e_{r r}(r)$ and $e_{\theta \theta}(r)$ at each of the 
measurement points gives the predicted values $e_{r r}^{j}=e_{r r}\left(r_{j}\right)$ and $e_{\theta \theta}^{j}=e_{\theta \theta}\left(r_{j}\right)$. In order to measure the goodness of the prediction, we form a functional $J$ given by the sum of squares of differences between actual measurements and the predicted values, with weights

$$
J=\sum_{j=1}^{m}\left[w_{r r}^{j}\left(\varepsilon_{r r}^{j}-e_{r r}^{j}\right)^{2}+w_{\theta \theta}^{j}\left(\varepsilon_{\theta \theta}^{j}-e_{\theta \theta}^{j}\right)^{2}\right]
$$

The choice of weights $w^{j}$ can be made on the basis of additional information available; for example, they could be chosen based on the accuracy of individual measurements being interpreted. However, in the present analysis we shall ascribe equal unit weights to all squared differences appearing in Eq. (16).

Minimization of functional $J$ provides a rational variational basis for selecting the most suitable model to match the measurements, in terms of the overall goodness of fit. Given a set of fixed measurements $\varepsilon_{r r}^{j}$ and $\varepsilon_{\theta \theta}^{j}, J$ can be thought of as a function of the residual stress state, or any set of parameters that describes its generation.

One possibility is to use eigenstrains to define the residual stress and residual elastic strain state. These are inelastic strains of any origin that are responsible for producing residual stress. In principle, a continuous distribution of eigenstrains must be described by (at worst) three-dimensional variation of six scalars (or one symmetric second-rank tensor). One way to reduce the complexity of the problem is to represent the unknown source eigenstrain distribution as a linear combination, i.e., a truncated sum of basis functions with unknown coefficients

$$
\varepsilon^{*}(x)=\sum_{i=1}^{N} c_{i} \xi_{i}(x)
$$

Here, $N$ is the total number of basis distributions used in the model.

Because of the linearity of the direct problem, the predicted values $e_{j}$ of the residual elastic strain due to the eigenstrain distribution $\varepsilon^{*}(x)$ of Eq. (14) can themselves be written in the form of a superposition of responses to the basis eigenstrain distributions

$$
e_{j}=\sum_{i=1}^{N} c_{i} E_{i}\left(x_{j}\right)=\sum_{i=1}^{N} c_{i} e_{i j}
$$

with the same coefficients $c_{i}$ as in Eq. (17).

The inverse problem of determining the unknown eigenstrain distribution $\varepsilon^{*}(x)$ can then be reduced to the problem of determination of $N$ unknown coefficients $c_{i}$ that deliver a minimum to the functional $J$ in Eq. (16) [11]. Because of the linearity of Eq. (18) in terms $c_{i}, J$ is quadratic and positive definite in the unknown coefficients $c_{i}$. It follows that the functional has a unique minimum that is found by satisfying the condition

$$
\nabla_{c} J=0 \quad \text { or } \quad \frac{\partial J}{\partial c_{i}}=0, \quad i=1, \ldots, N
$$

This system of equations is linear in $c_{i}$. Therefore, the solution for the unknown coefficients $c_{i}$ can be readily found without iteration by inverting the linear system arising in Eq. (20).

Alternatively, instead of the unknown eigenstrains, $J$ can be thought of as a function of problem parameters $c$ and $\sigma_{Y}$. In the present paper, we focus our attention on this case.

\section{Solution of the Inverse Problem}

Before we undertake the solution of the inverse problem about the determination of parameters $c$ and $\sigma_{Y}$ by minimization of the mismatch between observed residual elastic strains and the elastoplastic model prediction, we consider the dependence of residual stresses, and hence strains, on these parameters.

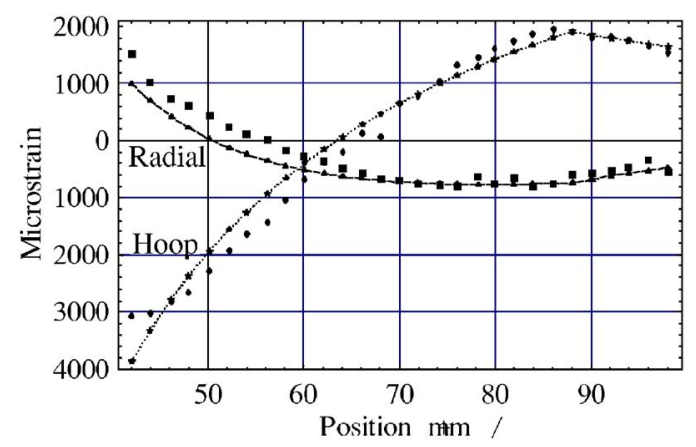

Fig. 7 Illustration of the shape of the surface defined by the function $J=J\left(c, \sigma_{Y}\right)$ over the $\left(c, \sigma_{Y}\right)$ plane

It can be readily established from the consideration of formulas of Sec. 2 that all residual stresses, both in the elastic and plastic regions, display linear dependence on the material yield stress $\sigma_{Y}$. Hence all residual strains must obey a similar linear dependence. This leads to $J$ being quadratic in $\sigma_{Y}$, guaranteeing the existence and uniqueness of solution (minimum) in terms of this parameter.

The dependence of residual stresses (and strains) on $c$ is more complex, since the expressions are not linear in $c$, e.g., Eq. (5). Furthermore, implicit dependence of $J$ on the position of the elastic-plastic boundary must be noted. It follows that the search for the solution may not be accomplished in "one shot" by inverting a linear system of equations, but may instead require the application of some nonlinear optimisation algorithm, such as steepest descent method, etc.

Instead of pursuing this kind of general analysis further, we focused our attention on some specific experimental data that we wished to interpret by employing inverse elastoplastic modeling. To this end we implemented the formulas of Sec. 2 in MATHEMATICA, in a way that ultimately allowed the mismatch functional $J$ to be written as a function of parameters $c$ and $\sigma_{Y}$, i.e., $J=J\left(c, \sigma_{Y}\right)$. Minimization of this function and the determination of the arguments $c^{*}$ and $\sigma_{Y}^{*}$ that deliver that minimum is readily achieved using the command FINDMINIMUM.

Figure 7 illustrates the shape of the surface defined by the function $J=J\left(c, \sigma_{Y}\right)$ above the $\left(c, \sigma_{Y}\right)$ plane. It is apparent that the dependence of $J$ on both those parameters is, in fact, not far from being perfectly quadratic, making the determination of the unique minimum a straightforward procedure.

\section{Results and Discussion}

The problem considered in the present paper is well known because of its simplicity and the availability of analytical solutions for stresses and strains in both elastic and plastic regions. It thus lends itself naturally to the consideration of the more difficult inverse problem of residual strain analysis, namely, the determination of the problem parameters (e.g., material yield stress or permanent strain distribution) that give rise to the observations conducted on the unloaded test piece.

The results of variational determination of the problem parameters are illustrated in Fig. 6, in which the markers indicate the values of residual elastic hoop and radial strains, respectively, as measured and reported by Venter et al. [8], whereas the continuous curves give the model predictions.

The agreement between the model and measurements is generally good-in fact, on the basis of the analysis presented in this paper it is possible to argue that it is the best that can be achieved with the elastic-ideally plastic model. On the other hand, the discrepancies between the model and measurements suggest that this simplistic model is incapable of capturing all complex hardening 
processes that occur within the workpiece due to autofrettage treatment. This issue has also been the subject of a study by Venter et al. [8].

In the present paper, we do not attempt to address this discrepancy. It is clear that a more sophisticated model with a larger number of parameters can provide greater flexibility and lead to a closer match to the experimental observations. The significant element of progress reported in the present study lies in the rational procedure that allows numerical determination of the best values of the system parameters to achieve this match.

\section{References}

[1] Lamé, G., 1852, Leçons sur la Théorie de l'Élasticité, Gauthier-Villars, Paris.

[2] Den Hartog, J. P., 1949, Strength of Materials, Dover, New York.

[3] Hill, R., 1950, The Mathematical Theory of Plasticity, Clarendon Press, Oxford.

[4] Soutas-Little, R. W., 1973, Elasticity, Dover, New York.

[5] Gao, X-L., and Wei, X.-X., 1991, "An Elasto-Plastic Analytical Solution for a
Closed-End Thick-Walled Cylinder of a Strain Hardening Material," Petrochem. Equip., 20, pp. 37-40.

[6] Gao, X-L., 1992, "An Exact Elasto-Plastic Solution for an Open-Ended ThickWalled Cylinder of a Strain-Hardening Material,” Int. J. Pressure Vessels Piping, 52, pp. 129-144.

[7] Gao, X-L., 2003, "Elasto-Plastic Analysis of an Internally Pressurized ThickWalled Cylinder Using a Strain Gradient Plasticity Theory," Int. J. Solids Struct., 40, pp. 6445-6455.

[8] Venter, A. M., de Swardt, R. R., and Kyriacou, S., 2000, "Comparative Measurements on Autofrettaged Cylinders With Large Bauschinger Reverse Yielding Zones," J. Strain Anal. Eng. Des., 35, pp. 459-469.

[9] Sachs, G., 1927, "Der Nachweis Inner Spannungen in Stangen und Rohren," Z. Metallkd., 72, pp. 352-357.

[10] Cheng, W., and Finnie, I., 1986, "Measurement of Residual Hoop Stresses in Cylinders Using the Compliance Method," ASME J. Eng. Mater. Technol., 108, pp. 87-92.

[11] Korsunsky, A. M., 2005, "On the Modelling of Residual Stresses Due to Surface Peening Using Eigenstrain Distributions," J. Strain Anal. Eng. Des., 40(8), pp. 817-824.

[12] Korsunsky, A. M., Regino, G. M., Nowell, D., and Liu, J., 2005, "Synchrotron Measurement and Eigenstrain Modelling of Residual Stress in Machined Nickel Alloy Plates," J. Strain Anal. Eng. Des., 41(5), pp. 381-395. 